\title{
Zur Kenntnis der Hydrazone und Azine
}

\author{
Erster Teil \\ Gefärbte Ketazine \\ Von \\ Otto Gerhardt
}

Aus dem Chemischen Laboratorium der Hochschule für Bodenkultur

(Vorgelegt in der Sitzung am 22. Jänner 1920)

Aldazine und Ketazine, die Endprodukte der Einwirkung von Hydrazin auf Aldehyde und Ketone, bilden sich mit stark voneinander verschiedener Geschwindigkeit aus den Komponenten. Die Aldazine werden meist erhalten aus äquimolekularen Mengen von Aldehyd und Hydrazinhydrat, bei den Ketonen hingegen bleibt die Reaktion mit Ausnahme der aliphatischen Ketone beim Hydrazon stehen. Die aromatischen Ketazine lassen sich nur durch Oxydation des Aminowasserstoffes mit Jod oder durch Erhitzen des gebildeten Hydrazons mit einem weiteren Molekül Keton darstellen.

Von aliphatischen Aldehyden und Ketonen wurden bisher relativ wenige Azine untersucht, die aromatischen Azine haben neutralen Charakter und sind in der Aldehydreihe ebenso wie die rein aliphatischen Vertreter leicht zerlegbar, in der Ketonreihe jedoch relativ sehr beständig.

Da sie im Molekül die chromophore Gruppe Azomethylen $>\mathrm{C}=\mathrm{N}-$ in gedoppelter Form enthalten, sind ihre aromatisch substituierten Vertreter meist gefärbt. Nun ist Azomethylen ebenso wie Carbonyl ein unselbständiger Chromophor und muß daher trotz seines gepaarten Auftretens nicht in allen 
Fällen Eigenfarbe erzeugen. Zum Auftreten einer solchen ist in diesem Falle oft noch das Vorhandensein einer auxochromen Gruppe oder auch besonders dichte Lagerung der Atome im Molekül notwendig.

Diese Tatsache bietet auch eine Erklärung für den Umstand, daß Benzophenonketazin (siehe die folgende Tabelle) farblos ist, indes das um zwei Phenylgruppen ärmere Benzaldazin goldgelb, sowie Acetophenon- und das gemischte Ketazin aus Benzophenon und Azeton, das eine hellgelb, das andere grünlich-gelb gefärbt sind. Daß das BenzophenonKetazin andrerseits doch chromogene Eigenschaften zeigt, erweist sich an dem Azin aus Michler's Keton, das kräftig braunrot gefärbt ist und dessen Lösungen schwach tingierende Eigenschaften zeigen.

Andrerseits wirkt Benzophenon farberhöhend, wenn es an die Stelle eines Benzilkomplexes im Benzilketazin tritt, wie sich an dem aus Benzil und Benzophenonhydrazon erhältlichen gemischten Azin erweist, das heller gelb gefärbt ist als das Benzilketazin. Durch Eintritt der auxochromen $-\mathrm{N}=\left(\mathrm{CH}_{3}\right)_{2}{ }^{-}$ Gruppe wird die Farbe ins Chromgelbe vertieft, wie das aus dem Hydrazon von Michler's Keton und Benzil erhältliche Azin beweist.

Gar nicht hingegen stimmt das Verhalten der substituierten Benzaldazine mit den Regeln der Auxochrom- und Chromophortheorie überein, denn das Azin des Salizylaldehyds (J. p., 39, p. 48) ist farblos, obwohl es eine auxochrome Hydroxylgruppe enthält, das Piperonalazin indes wieder schwach gelb, obwohl auxochrome Eigenschaften der am Benzolring haftenden Oxymethylengruppe bisher nicht bekannt waren. Es finden sich somit in den Beziehungen zwischen Farbe und Konstitution der Azine keine strengen Gesetzmäßigkeiten.

Besonders auffallend ist unter den Azinen dasjenige des Fluorenons, das tiefrote Eigenfarbe zeigt; an ihm zeigt sich der Einfluß der dichteren Lagerung der Atome im Molekül, aber außerdem ergibt sich aus der Betrachtung seines Formelbildes, daB in ihm bei Annahme der Kekule'schen Benzolformel ein System gekreuzter Doppelbindungen auftritt, die ebenso wie in den Fulvenen neben dem Vorhandensein der 
Azomethylengruppe und der dichteren Lagerung die einander verstärkenden Ursachen für die tiefe Eigenfarbe des Azins sind:

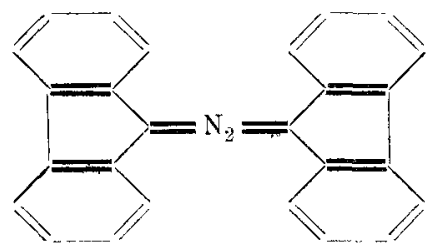

In Übereinstimmung mit der eben dargelegten Anschauung ist das Ketazin des $\beta$-Hydrindons,

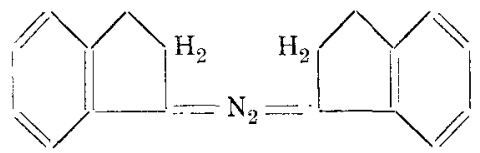

da bei sonst sehr ähnlicher Konstitution die gekreuzten Doppelbindungen fehlen, bloß schwach rosenrot gefärbt, ebenso wie das schon länger bekannte Alpha-Hydrindonketazin (Soc., 71, p. 250), das schwach gelblich gefärbt ist.

Der Eintritt auxochromer Gruppen in das Moleküi dieses Ketazines ließ nun Farbvertiefung, wahrscheinlich auch Farbstoff bildung erwarten; um letztere Frage zu entscheiden, bedurfte es allerdings noch der Herstellung wasserlöslicher Derivate, um die erhaltenen Körper auf ihre tingierenden Eigenschaften $z u$ prüfen. Letzteres gelang indes nicht,' da sulfo- oder carbonsaure Salze der Ketazine weder direkt noch indirekt erhältlich waren.

Es ist nun bereits eine beträchtliche Anzahl amido- und auch oxysubstituierter Fluorenone bekannt. Von diesen waren alle jene für den $Z$ weck der vorliegenden Untersuchung tauglich, welche die Amido-, beziehungsweise Oxygruppe in der die Farbvertiefung, beziehungsweise Farbstoffbildung begünstigenden Meta- oder Parastellung zur chromophoren $>\mathrm{C}=\mathrm{O}$-, beziehungsweise $>\mathrm{C}=\mathrm{N}$-Gruppe enthielten, somit 2-, 3- oder 4-Amino-, beziehungsweise Oxyfluorenon (siehe nachfolgende Formel). Dargestellt hiervon waren bislang 2-Aminofluorenon von $\mathrm{Diels}$ (Ber., 34, p. 1758 ff.), 2-Oxyfluorenon (derselbe) und 4-Aminofluorenon von Graebe und Schestakow (Ann., 284, 
p. $310 \mathrm{ff}$.). Von 3-substituierten Fluorenonen war bisher bloß das 3-Nitrofluorenon bekannt, dargestellt von Schmidt (Ber., 41, p. 3691 ff., beziehungsweise C., 1908, II, 1870), aus dem durch Reduktion 3-Aminofluorenon herzustellen leicht möglich erschien.

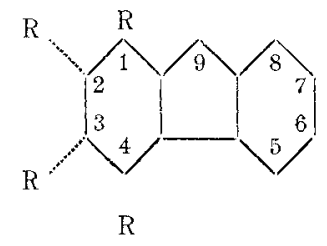

Bei der vorliegenden Untersuchung wurden bloß die direkt vom Fluoren aus zugänglichen Derivate angewandt, da ebenso 3-Nitro-, wie 4-Aminofluorenon die Anwendung von unter den herrschenden Verhältnissen in genügenden Mengen nicht erreichbaren Ausgangsmaterialien, wie Phenanthrenchinon (1. c.) und Hydroxylaminhydrochlorid (1. c.) erforderten.

Diels (1. c.) war durch Oxydation des aus Fluoren leicht erhältlichen 2-Nitrofluorens

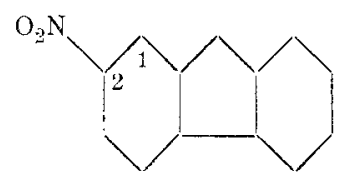

mit Eisessig-Chromatgemisch zum 2-Nitrofluorenon unter Erzielung fast quantitativer Ausbeute gelangt und hatte aus diesem durch Reduktion mit Schwefelwasserstoff in alkoholischammoniakalischer Lösung 2-Aminofluorenon in 60-prozentiger Ausbeute gewonnen, einen tiefroten Körper, der indes noch keine tingierenden Eigenschaften zeigt.

Stellt man nun zunächst aus 2-Nitrofluorenon das Hydrazon und aus diesem durch Oxydation mit Jod das Ketazin dar, so erhält man dieses als ein hellbraun gefärbtes Pulver, das in Lösung ebensolche Farbe zeigt. In diesem Falle tritt also Farberhöhung ein, eine Tatsache, die indes mit der vorliegenden Frage nicht im direkten Zusammenhange steht, da die Nitrogruppe stets als Chromophor auftritt. Immerhin aber ist ihre hier farberhöhende Wirkung als interessant zu vermerken. 
Vom Nitrofluorenon ausgehend, wurde nun weiterhin versucht, das Ketazin des 2-Aminofluorenons, beziehungsweise des Dimethyl-2-Aminofluorenons darzustellen, um jenes auf Eigenfarbe sowie auf das eventuelle Auftreten tingierender Eigenschaften in Substanz, beziehungsweise bei seinen Derivaten $z u$ prüfen. 2-Aminofluorenonhydrazon läßt sich wegen der ungeschützten Aminogruppe nicht mit Jod $z u$ dem entsprechenden Ketazin oxydieren. Es wurde daher versucht, durch Erhitzen von 1 Mol Hydrazon mit einem weiteren Mol Keton unter Druck zum Ketazin zu gelangen. Auch dies erwies sich als nicht durchführbar, da bei Durchführung der Reaktion stets Verschmierung eintrat. Es wurde daher die Aminogruppe im 2-Aminofluorenon durch Einführung einer Acetylgruppe geschüitzt und das davon sich ableitende Acetyl-2-Aminofluorenonhydrazon der Oxydation mit Jod unterworfen. Es resultierte hierbei hellbraunes Acetyl-2-Aminofluorenonketazin, das indes auffallenderweise durch verseifende Mittel nicht zu spalten war, denn mittelstarkes alkoholisches Kali (doppeltnormal) wirkt nicht ein, stärkstes (zirka 5-normal) verseift wohl, doch wirkt es, wie zu erwarten, gleich auf das gebildete 2 -Aminofluorenonketazin verharzend ein, so daß sich dieses auf diese Weise nicht isolieren läßt.

Da sich nun so die Unmöglichkeit ergab, zum 2-Aminofluorenonketazin zu gelangen, wurden in die Aminogruppe des Ketons zwei Methylgruppen eingeführt und versucht, weiterhin aus dem so entstandenen Dimethyl-2-Aminofluorenon zum Hydrazon und Ketazin zu gelangen. Dies scheiterte indes schon an dem Umstand, daß die Hydrazonbildung bei dem Dimethylprodukt nicht eintrat, denn beim Erhitzen von Dimethyl2-Aminofluorenon mit der berechneten Menge Hydrazinhydrat in alkoholischer Lösung trat stets weitgehende Verharzung ein und es konnte kein definierter Körper aus dem Reaktionsprodukt isoliert werden. Analogien für dieses Verhalten finden sich, denn es läßt sich z. B. das Hydrazon des Dimethylaminobenzils ebenfalls nur in sehr schlechter Ausbeute unter starker Verschmierung darstellen.

Die Hoffnung, zu aminosubstituiertem Fluorenonketazin zu gelangen, erwies sich somit a1s trügerisch, hingegen konnte 
noch das Ketazin des 2-Oxyfluorenons (Diels, I. c.) erhalten werden, was auch glatt gelang, denn ebensowohl das Hydrazon als auch das Ketazin entstanden bei den entsprechenden Reaktionen in guter Ausbeute. Es stand nun für 2-Oxyfluorenonketazin eine deutliche Farbvertiefung gegenüber dem nicht substituierten Grundkörper zu erwarten: diese war indes nicht deutlich eingetreten, denn 2-Oxyfluorenonketazin ist in Substanz sowie in Lösung braun gefärbt. Auch konnten mit ihm keine haltbaren Färbungen auf pflanzlicher oder tierischer Faser erzeugt werden. Sulfosäurederivate des Ketazins, die eine eingehende Prüfung auf die Verwandtschaft zur Faser gestattet hätten, darzustellen, erwies sich als unmöglich: es konnte somit die Frage nach der Farbstoffnatur des Ketazins nicht mit voller Bestimmtheit beantwortet werden.

Durch die vorstehend gebrachten Tatsachen erweist sich also, daß auch bei dem Fluorenonketazin strenge Gesetzmäßigkeiten in den Beziehungen zwischen Farbe und Konstitution nicht auftreten; im Gegenteil ergibt sich sogar, daß trot $z$ Anwesenheit der chromophoren Gruppe $>\mathrm{C}=\mathrm{N}-$, trotz der dichten Lagerung der Atome im Molekül, sowie trotz dem Auftreten gekreuzter Doppelbindungen durch den Eintritt der auxochromen Hydroxylgruppe in Metastellung nur unerhebliche Farbvertiefung, anscheinend aber keine Farbstoffbildung resultiert.

\section{Experimenteller Teil.}

\section{Beta-Hydrindonketazin.}

$20 \mathrm{~g}$ (2 Mol) trisch hergestelltes Beta-Hydrindon (Ber., 32, p. 28) vom F. $58^{\circ}$ unkorr. Werden in $40 \mathrm{~cm}^{3}$ Alkohol auf dem Wasserbade gelöst und nach dem Erkalten mit $8 g(1 \mathrm{Mol})$ Hydrazinhydrat versetzt. Es tritt starke Erwärmung ein und das Reaktionsprodukt fällt als gelbe Krystalimasse aus. Nach Abkühlen unter der Wasserleitung wird abgesaugt und mit wenig Alkohol nachgewaschen. Zum Umkrystallisieren wird die krümmelige Krystallmasse nach dem Trocknen grob gepulvert und mit viel Benzol mehrmals ausgekocht. Beim Abkühlen fallen aus der filtrierten Lösung blaßrosa gefärbte 
Krystallnadeln des Ketazins aus, die bei 194 bis $195^{\circ}$ (unkorr:) unter Zersetzung schmelzen. Die Ausbeute ist quantitativ, jedoch empfiehlt es sich nicht, das Auskochen mit Benzol mehr als dreimal $\mathrm{zu}$ wiederholen, wenn auch dadurch die Ausbeute an reinem Produkt sinkt, denn man beobachtet beim Auskochen des Rohproduktes die Abscheidung tiefrot gefärbter Krusten an der Grenzlinie zwischen Lösung und Glaswand des Rundkolbens, ebenso bei öfters wiederholtem Auskochen immer tiefere Färbung der Lösung und schließlich krystallisieren ziemlich tiefrote Nadeln von tieferem Schmelzpunkt als dem der reinen Substanz aus.

Das Ketazin ist schwer löslich in kaltem und heißem Alkohol, ebenso in kaltem Benzol. In heißem Benzol ist es ziemlich reichlich löslich, weshalb sich dieses Lösungsmittel am besten zum Umkrystallisieren eignet. Pyridin löst leicht, doch erhält man daraus stets tiefrot gefärbte, unreine Produkte. Die Ursache für diese Rotfärbung liegt in der Leichtigkeit, mit der das Ketazin sich autoxydiert, denn schüttelt man z. B. eine Suspension von feingepulvertem Ketazin in trockenem Benzol mehrere Tage unter Feuchtigkeitsabschluß mit Luft, so geht dieses allmählich unter Bildung tiefroter Körper in Lösung. Versuche, aus diesen tiefgefärbten Autoxydationsprodukten definierte Körper $\mathrm{zu}$ isolieren, hatten keinen Erfolg und es wurde die weitere Verfolgung dieser vorläufigen Versuchsergebnisse einem späteren Zeitpunkte vorbehalten.

Beta-Hydrindonketazin zeigt mit konzentrierter Schwefelsäure blaugrüne Halochromie, wie sie für alle Indenverbindungen charakteristisch ist.

Analysen:

$5 \cdot 470 \mathrm{mg}$ ergaben $16 \cdot 705 m g \mathrm{CO}_{2}, 2 \cdot 99 \mathrm{mg}_{\mathrm{s}} \mathrm{H}_{2} \mathrm{O}$.

$4.012 \mathrm{mg}$ ergaben $0.382 \mathrm{~cm}^{3} \mathrm{~N}_{2}$ bei $732 \mathrm{~mm}, 16^{\circ} \mathrm{C}$.

Gef. $83 \cdot 130 \% \mathrm{C}$; ber. f. $\mathrm{C}_{18} \mathrm{H}_{16} \mathrm{~N}_{2}: 83 \cdot 030 \% \mathrm{C}$;

$6.11 \% \mathrm{H} ; \quad 6.07 \% \mathrm{H}$

$10 \cdot 830 \% \mathrm{~N} . \quad 10 \cdot 76 \% \mathrm{~N}$.

2-Nitrofluorenonhydrazon.

Da das 2-Nitrofluorenon schwer löslich in Alkohol ist, nimmt man vorteilhaft die Darstellung des Hydrazons im 
Bombenrohr vor: $1.6 \mathrm{~g}$ Keton werden mit $0.5 \mathrm{~g}$ Hydrazinhydrat und $3 \mathrm{~cm}^{3}$ Alkohol 6 Stunden im Einschlußrohr auf $85^{\circ}$ erhitzt. Das braungelbe Reaktionsprodukt wird aus viel siedendem Alkohol umkrystallisiert, wobei das Hydrazon in dunkelgelben, mikroskopischen Blättchen vom F. $214^{\circ}$ erhalten wird. Ausbeute $90 \%$ der Theorie. Zur Analyse wurde nochmals aus viel Alkohol umkrystallisiert.

Der Körper ist schwer löslich in Alkohol und den meisten organischen Lösungsmitteln, leicht löslich hingegen in Azeton, woraus er indes nur verschmiert auskrystallisiert.

Analysen:

$2.250 \mathrm{mg}^{2}$ ergaben $0.362 \mathrm{~cm}^{3} \mathrm{~N}, \mathrm{~b}$ bei $738 \mathrm{~mm}, 24^{\circ} \mathrm{C}$.

Gef. $17 \cdot 690 / 0$ N. Ber, für $\mathrm{C}_{13} \mathrm{H}_{9} \mathrm{O}_{2} \mathrm{~N}_{3}: 17 \cdot 570 / 0 \mathrm{~N}$.

\section{2-Nitrofluorenonketazin.}

Infolge der oben erwähnten Schwerlöslichkeit des 2-Nitrofluorenonhydrazons in den gebräuchlichen Lösungsmitteln grestaltet sich die Darstellung des Ketazins durch Oxydation mit Jod mißlich. Es wurde daher auch versucht, durch Erhitzen von 1 Mol Hydrazon mit $1 \mathrm{Mol} 2$-Nitrofluorenon unter Beigabe von etwas Alkohol im Druckrohr zum Ketazin zu gelangen. Die Reaktion geht aber hier bei Temperaturen bis $160^{\circ} \mathrm{zu}$ langsam vor sich. Bei $180^{\circ}$ hingegen verschmiert das Reaktionsgemisch und so mußte das Ketazin doch durch Oxydation des Hydrazons mit Jod unter mehrstündigem Kochen in alkoholischer Lösung dargestellt werden. Am besten verfährt man hierbei folgendermaßen:

$4 g$ 2-Nitrofluorenonhydrazon werden feinst gepulvert in $150 \mathrm{~cm}^{3}$ Alkohol suspendiert und in die am Rückfluß kochende Flüssigkeit im Verlaufe von 2 Stunden $16 \mathrm{~g}$ Jod (2 Mole) in kleinen Partien eingetragen. Nachher wird noch zirka eine halbe Stunde gekocht und nach dem Abkiihlen das in Form eines braunen, mikrokrystallinischen Pulvers ausgefallene Ketazin abgesaugt. Durch mehrmaliges Auskochen mit Alkohol werden Reste unangegriffenen Hydrazons entfernt und schließlich aus viel siedendem Xylol umkrystallısiert. Man erhält so ein braunes Krystallpulver vom F. 305 bis $306^{\circ}$ C., schwer 
löslich in allen organischen Lösungsmitteln. Ausbeute $60 \%$ der Theorie. Mit konzentrierter Schwefelsäure gibt das Ketazin braunrote Halochromie.

Analyse:

$2 \cdot 79 \mathrm{mg}$ ergaben $0.3047 \mathrm{~cm}^{3} \mathrm{~N}_{2}$ bei $738 \mathrm{~mm}, 19^{\circ} \mathrm{C}$.

Gef. $12 \cdot 40 \%$ N. Ber. für $\mathrm{C}_{26} \mathrm{H}_{14} \mathrm{O}_{4} \mathrm{~N}_{4}: 12 \cdot 56 \% \mathrm{~N}$.

\section{2-Aminofluorenonhydrazon.}

$1.5 \mathrm{~g} 2$-Aminofluorenon, $0.4 \mathrm{~g}$ Hydrazinhydrat und $7 \mathrm{~cm}^{3}$ Alkohol werden 1 Stunde auf dem Wasserbad erwärmt. Beim Erkalten scheidet sich das Hydrazon in orangegelben, verfilzten Krystallnädelchen vom F. 201 bis $202^{\circ}$ ab. Ausbeute $1.38 g$ $=75 \%$ der Theorie. Zur Analyse wurde aus heißem Alkohol zweimal umkrystallisiert.

Analyse:

$3.024 \mathrm{mg}$ ergaben $0.538 \mathrm{~cm}^{3} \mathrm{~N}_{2}$ bei $750 \mathrm{~mm}, 21^{\circ} \mathrm{C}$.

Gef. $20 \cdot 200 \%$ N. Ber, für $\mathrm{C}_{13} \mathrm{H}_{11} \mathrm{~N}_{3}: 20 \cdot 090_{00} \mathrm{~N}$.

\section{Acetyl-2-Aminofluorenon.}

$2 g$ 2-Aminofluorenon werden mit $2 \mathrm{~cm}^{3}$ Essigsäureanhydrid 15 Minuten in einem kleinen Kölbchen zum Sieden erwärmt und nach dem Erkalten das ausgeschiedene Acetylprodukt abgesaugt. Ausbeute quantitativ. Das leuchtend zinnoberrote Keton schmilzt nach einmaligem Umkrystallisieren aus Alkohol scharf bei 227 bis $228^{\circ}$ und ist bereits analysenrein.

Analysen:

$2 \cdot 468 m g$ exgaben $6 \cdot 885 m_{\mathrm{S}} \mathrm{CO}_{2}, 1 \cdot 078 m g \mathrm{H}_{2} \mathrm{O}$.

Gef. $76 \cdot 08 \%$ C, $4 \cdot 900 \% \mathrm{H}$.

$3.782 \mathrm{mg}^{\circ}$ ergahen $0.204 \mathrm{~cm}^{3} \mathrm{~N}_{2}$ bei $748 \mathrm{~mm}, 22^{\circ} \mathrm{C}$.

Gef. $6 \cdot 15 \% \mathrm{~N}$.

Ber. für $\mathrm{C}_{15} \mathrm{H}_{11} \mathrm{O}_{2} \mathrm{~N}: 75 \cdot 95 \% \mathrm{C}, \quad 4 \cdot 64 \% \mathrm{H}, \quad 5 \cdot 91 \% \mathrm{~N}$.

\section{Acetyl-2-Aminofluorenonhydrazon.}

$2 g$ Acetyl-2-Aminofluorenon, $0.4 g$ Hydrazinhydrat und $5 \mathrm{~cm}^{3}$ Alkohol werden eine halbe Stunde auf dem Wasserbad erwärmt. Das Hydrazon scheidet sich nach dem Erkalten in 
zitronengelben Blättchen vom F. 212 bis $214^{\circ}$ aus. Ausbeute quantitativ. Zur Analyse wurde zweimal aus Alkohol umkrystallisiert.

Analyse:

$2.910 \mathrm{mg}$ ergaben $0.441 \mathrm{~cm}^{3} \mathrm{~N}_{2}$ bei $738 \mathrm{~mm}, 22^{\circ} \mathrm{C}$.

Gef. $17 \cdot 03 \%$ N. Ber, für $\mathrm{C}_{15} \mathrm{H}_{13} \mathrm{ON}_{3}: 16 \cdot 73 \% \mathrm{~N}$.

\section{Acetyl-2-Aminofluorenonketazin.}

$2 \mathrm{~g}$ Acetyl.2-Aminofluorenonhydrazon wurden in $10 \mathrm{~cm}^{3}$ Alkohol gelöst und in der Siedehitze mit $8 g$ Jod oxydiert. Das abgeschiedene Ketazin wird nach dem Absaugen und Waschen aus sehr viel siedendem Xylol umkrystallisiert und schmilzt nach mehrmaligem Umkrystallisieren unscharf zwischen 303 und $306^{\circ}$ C. (unkorr.). Ausbeute $60 \%$ der Theorie. Es stellt ein braunes, in allen organischen Lösungsmitteln schwer lösliches Krystallpulver dar, das sich in konzentrierter Schwefelsäure mit brauner Farbe löst und durch Wasser, beziehungsweise Alkali daraus wieder unverändert gefällt wird. Analyse:

$2.52 \mathrm{mg}_{\mathrm{s}}$ ergaben $0.265 \mathrm{~cm}^{3} \mathrm{~N}_{2}$ bei $740 \mathrm{~mm}, 22^{\circ} \mathrm{C}$.

Gef. $11 \cdot 82 \%$ No. Ber. für $\mathrm{C}_{30} \mathrm{H}_{22} \mathrm{O}_{2} \mathrm{~N}_{1}: 11 \cdot 910 \%$ N.

\section{Versuche}

zur Verseifung des Acetyl-2-Aminofluorenonketazins.

1. $0.5 \mathrm{~g}$ Ketazin wurden mit $10 \mathrm{~cm}^{3}$ doppeltnormaler alkoholischer Kalilauge 2 Stunden am Rückfluß gekocht. Der ungelöste Körper erwies sich nach dem Waschen und Umkrystallisieren als unverändertes Ketazin.

2. $0.5 \mathrm{~g}$ Ketazin wurden mit $10 \mathrm{~cm}^{3}$ einer gesättigten alkoholischen Kalilauge eine halbe Stunde am Rückfluß gekocht. Nach dieser Zeit war das ursprünglich vorhandene Krystallpulver in schmierige, dunkle Massen umgewandelt, aus denen sich indes kein definierter Körper isolieren ließ.

\section{Dimethy1-2-Aminofluorenon.}

Die Methylierung des 2-Aminofluorenons erfolgt am besten nach der Methode von Ullmann (Ann., 327, p. 104, 120) 
mittels Dimethylsulfat, die von dem Genannten zur Einführung einer und auch zweier Methylgruppen in Phenole, beziehungsweise Amine ausgearbeitet wurde. Im hier angegebenen Falle wurde folgendermaßen verfahren:

$14 \mathrm{~g}$ 2-Aminofluorenon wurden im Verlauf zweier Stunden in $40 \mathrm{~g}$ Dimethylsulfat (zirka 2.3 Mole) eingetragen, die im Ölbade auf einer Temperatur von 170 bis $180^{\circ} \mathrm{C}$. gehalten wurden und nach dem vollständigen Eintragen noch 1 Stunde bei dieser Temperatur belassen. Nach dem Abkühlen wurde in einen Überschuß Natronlauge eingetragen und zur Zerstörung des überschüssigen Dimethylsulfats aufgekocht, vom Ungelösten abgesaugt und dieses mehrmals aus wenig heißem Alkohol umkrystallisiert. Das reine Dimethylaminofluorenon, das in 40-prozentiger Ausbeute erhalten wurde, ist ein tiefrotes Krystallpulver, dessen alkoholische Lösungen blaustichigrot gefärbt sind, zum Unterschiede von denen des Aminofluorenons, die ein zwar dunkles, aber reines Rot zeigen. Die reine Substanz schmilzt bei 146 bis $147^{\circ}$ C. (unkorr.).

Analyse:

$5.030 \mathrm{mg}$ ergaben $0.292 \mathrm{~cm}^{3} \mathrm{~N}_{2}$ bei $740 \mathrm{~mm}, 22^{\circ} \mathrm{C}$.

Gef. $6 \cdot 54 \%$ N. Ber. für $\mathrm{C}_{15} \mathrm{H}_{13} \mathrm{ON}: 6 \cdot 28 \% \mathrm{~N}$.

\section{2-Oxyfluorenonhydrazon.}

$2 g$ Oxyfluorenon, $0.4 \mathrm{~g}$ Hydrazinhydrat und $5 \mathrm{~cm}^{3}$ Alkohol wurden eine halbe Stunde auf dem Wasserbad erwärmt. Das Hydrazon schied sich nach dem Erkalten in chromgelben, verfilzten Krystallnadeln vom F. 201 bis $202^{\circ}$ C. aus. Ausbeute quantitativ. Zur Analyse wurde zweimal aus Alkohol umkrystallisiert.

Analyse:

$2 \cdot 788 \mathrm{mg}^{3}$ ergaben $0.330 \mathrm{~cm}^{3} \mathrm{~N}_{2}$ bei $749 \mathrm{~mm}, 20^{\circ} \mathrm{C}$.

Gef. $13 \cdot 60 \%$ N. Ber. f. $\mathrm{C}_{13} \mathrm{H}_{10} \mathrm{ON}_{2}: 13 \cdot 34 \%$ N.

\section{2-Oxyfluorenonketazin.}

Das Ketazin wurde durch Oxydation des Hydrazons mit der berechneten Menge Jod in alkoholischer Lösung bei Siedehitze in 70-prozentiger Ausbeute 1eicht erhalten. Infolge seiner 
Schwerlöslichkeit in organischen Lösungsmitteln wurde es durch mehrmaliges Lösen in Alkali und Fällen mit Salzsäure gereinigt. Im reinen Zustande schmilzt es bei 301 bis $303^{\circ} \mathrm{C}$. und stellt ein braunes Krystallpulver dar, das in Alkohol löslich ist und sich in unverändertem Zustande durch Säure wieder ausfällen läßt. Mit Schwefelsäure gibt es braunrote Färbungen.

Analyse:

$3.454 \mathrm{mg}^{2}$ ergaben $0.226 \mathrm{~cm}^{3} \mathrm{~N}_{2}$ bei $749 \mathrm{~mm}^{\circ} 20^{\circ} \mathrm{C}$.

Gef. $7 \cdot 520,0$ N. Ber. für $\mathrm{C}_{26} \mathrm{H}_{16} \mathrm{O}_{2} \mathrm{~N}_{2}: 7 \cdot 220 \% \mathrm{~N}$.

\section{Piperonalaldazin.}

Das Aldazin wurde durch Versetzen einer alkoholischen Piperonallösung mit der berechneten Menge Hydrazinhydrat in der Kälte erhalten. Es fiel hierbei in schwachgelb gefärbten Krystallblättchen aus und war nach einmaligem Umkrystallisieren aus viel heißem Alkohol analysenrein. Ausbeute quantitativ, F. 206 bis $207^{\circ}$ C. Gelbe Halochromie mit konzentrierter Schwefelsäure.

Analyse:

$3.155 \mathrm{mg}$ ergaben $0.267 \mathrm{~cm}^{3} \mathrm{~N}_{2}$ bei $744 \mathrm{~mm}, 26^{\circ} \mathrm{C}$.

Gef. $9 \cdot 540 \%$ N. Ber. für $\mathrm{C}_{16} \mathrm{H}_{12} \mathrm{O}_{4} \mathrm{~N}_{2}: 9 \cdot 46 \%$ N.

\section{Benzophenon-Benzilketazin.}

$3 g$ Benzil, $2 \cdot 8 g$ Benzophenonhydrazon und $1 \mathrm{~cm}^{3}$ Alkohol wurden im Druckrohr 6 Stunden auf $150^{\circ} \mathrm{C}$. erhitzt. Nach dem Erkalten wurde der Rohrinhalt, eine gelbbraune Schmiere, durch Anreiben mit Petroläther und Äther zur Krystallisation gebracht, abgesaugt und mit dem erwähnten Gemische nachgewaschen. Das schwach gelb gefärbte Ketazin wurde durch zweimaliges Umkrystallisieren aus Alkohol als hellgelbe Krystallwarzen vom F. 128 bis $129^{\circ}$ C. erhalten. Ausbeute $66^{\circ} \%$ der Theorie, gelbe Halochromie mit Schwefelsäure.

Analyse:

$3.408 \mathrm{mg}$ ergaben $0.225 \mathrm{~cm}^{3} \mathrm{~N}_{2}$ bei $747 \mathrm{~mm}, 21^{\circ} \mathrm{C}$.

Gef. $7 \cdot 55 \%$ N. Ber. für $\mathrm{C}_{27} \mathrm{H}_{20} \mathrm{ON}_{2}: 7 \cdot 22 \% \mathrm{~N}$. 
Tetramethyldiamidobenzophenon-Benzilketazin.

$2 \cdot 1 \mathrm{~g}$ Benzil, $2 \cdot 84 \mathrm{~g}$ Hydrazon von Michler's Keton und $3 \mathrm{~cm}^{3}$ Alkohol wurden 6 Stunden im Einschlußrohr auf $150^{\circ} \mathrm{C}$. erhitzt. Das Reaktionsprodukt bildete nach dem Erkalten eine braungelbe, schmierige Masse, aus der durch Anreiben mit Petroläther und Äther das Ketazin in Form orangegefärbter Blättchen gewonnen wurde. Es wurde durch mehrmaliges Umkrystallisieren aus Alkohol gereinigt und schmolz im analysenreinen Zustande bei 173 bis $174^{\circ} \mathrm{C}$. Orangerote Färbung mit konzentrierter Schwefelsäure.

Analyse:

$2.402 \mathrm{mg}$ ergaben $0.252 \mathrm{~cm}^{3} \mathrm{~N}_{2}$ bei $751 \mathrm{~mm}, 21^{\circ} \mathrm{C}$.

Gef. $12 \cdot 02 \%$ N. Ber. für $\mathrm{C}_{31} \mathrm{H}_{30} \mathrm{ON}_{4}: 11 \cdot 81 \%$ N. 
o. Gerhardt,

Aldazine.

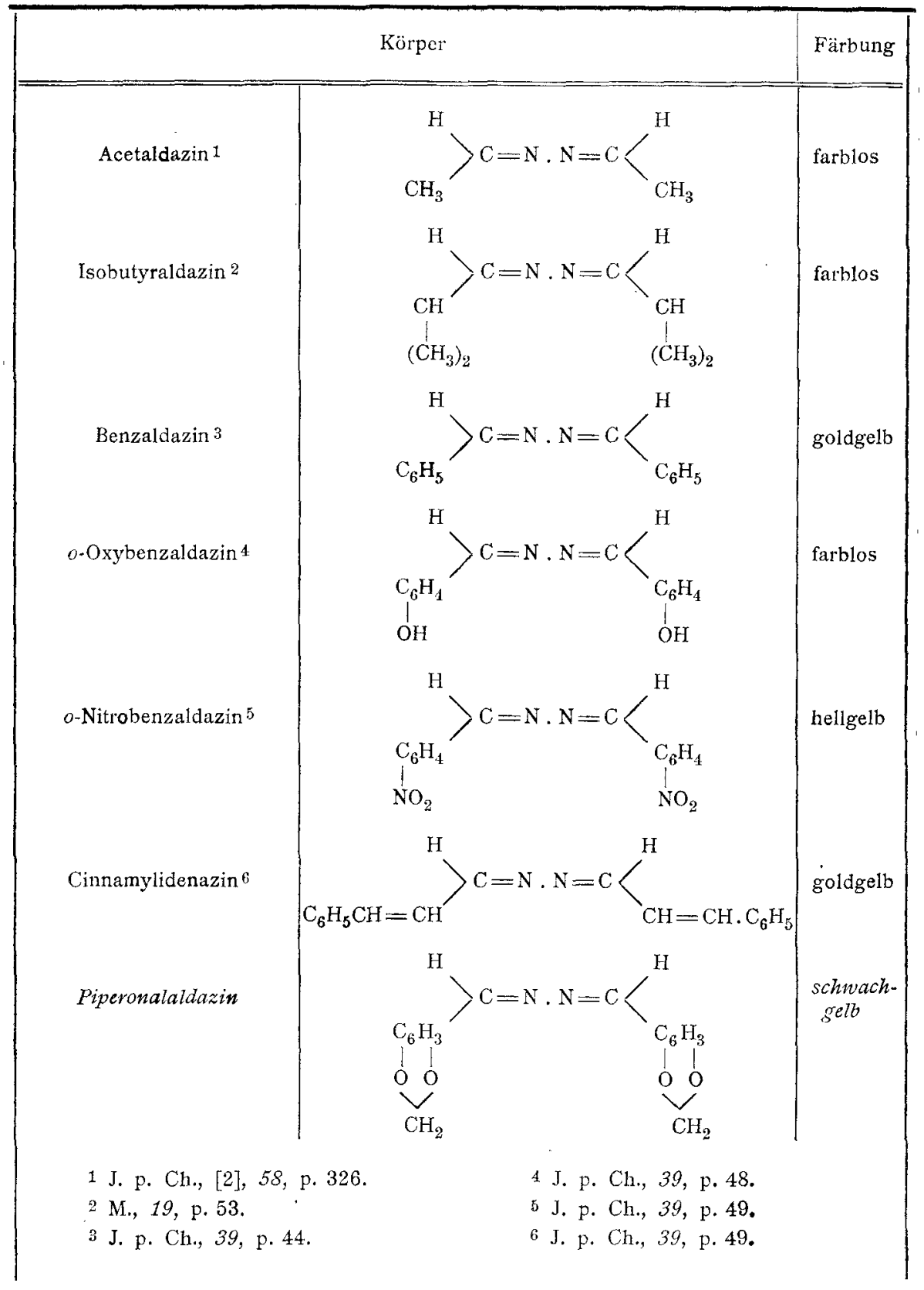




\section{Ketazine.}

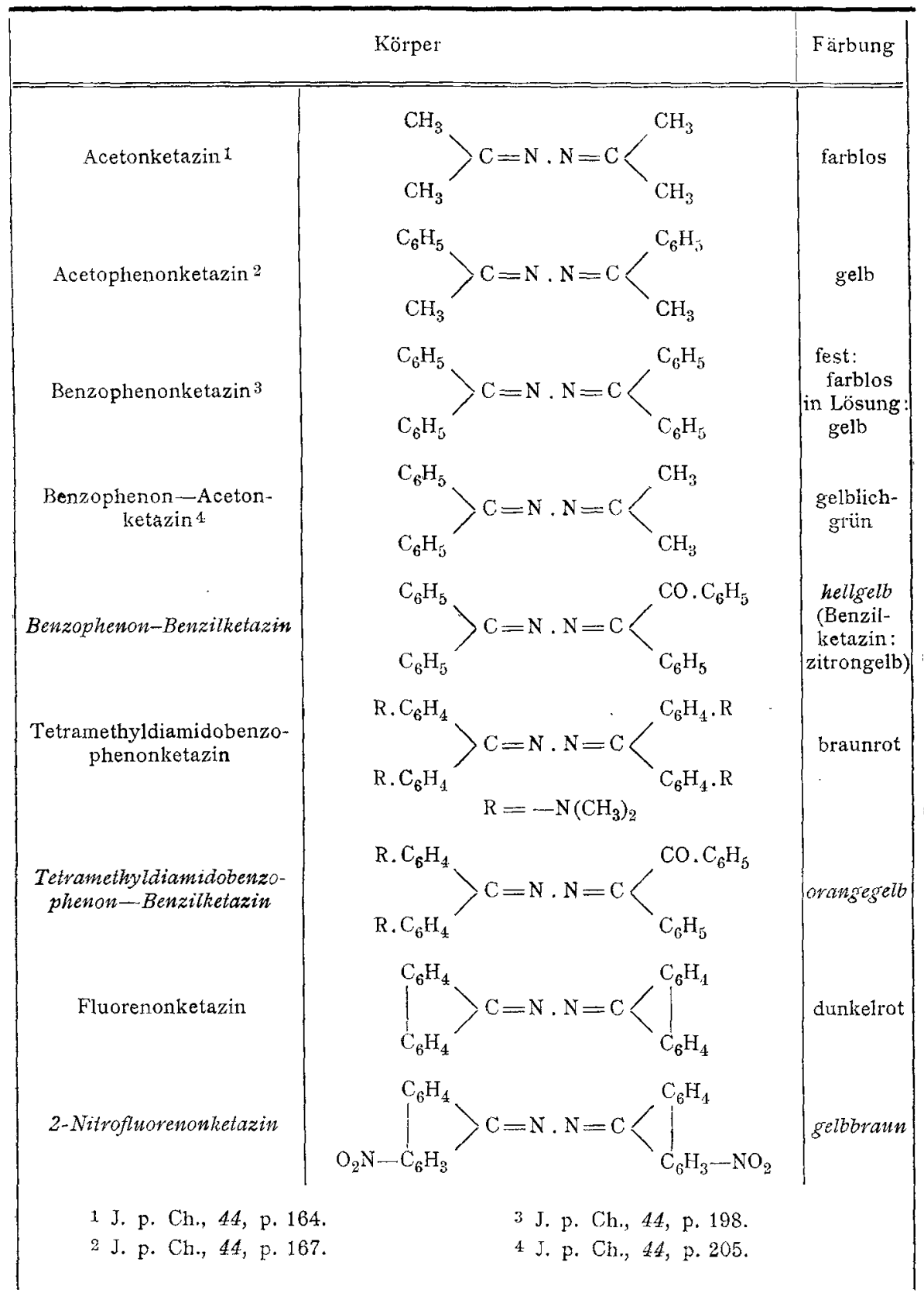




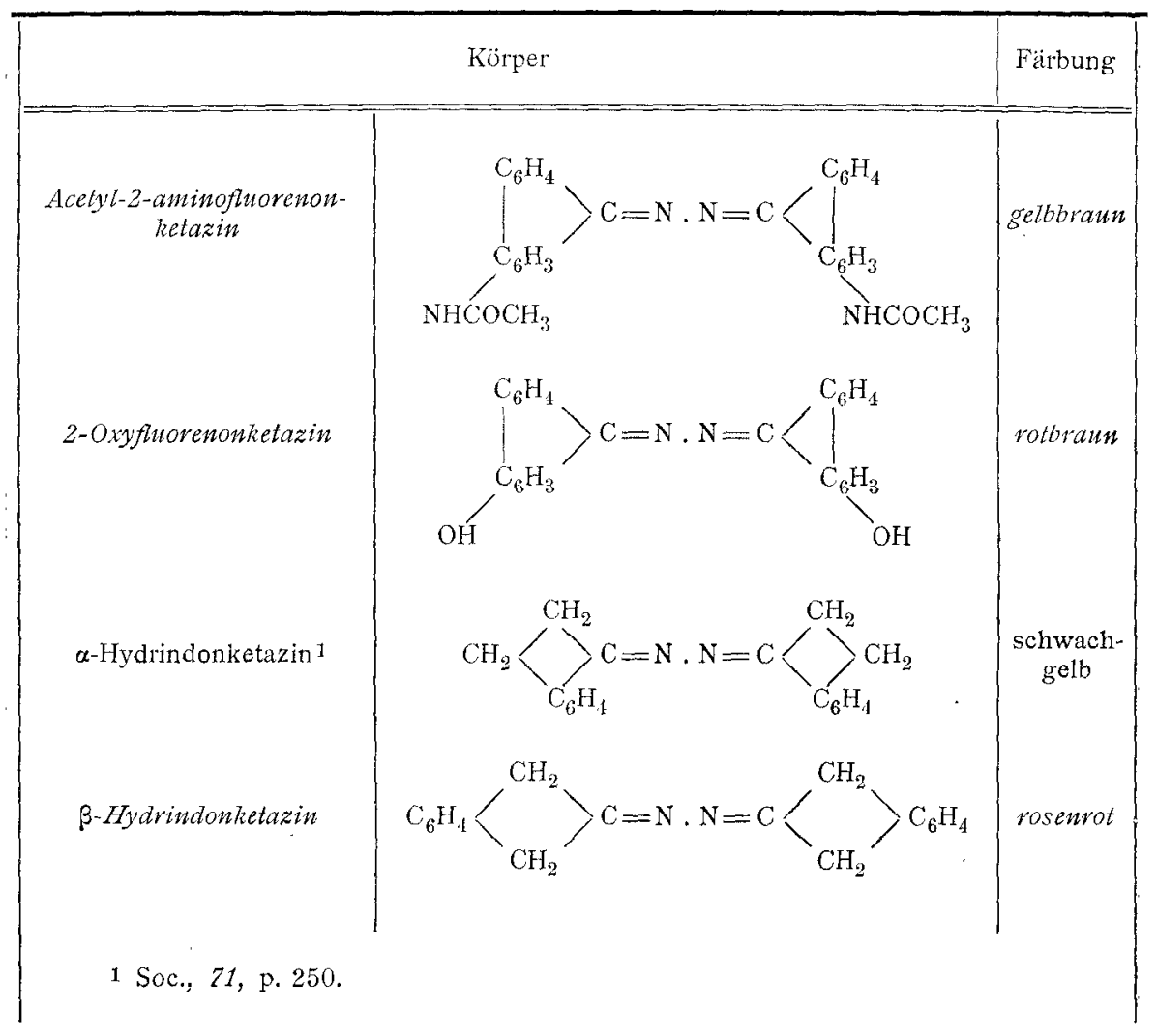

